Published in final edited form as:

Cancer Chemother Pharmacol. 2008 December ; 63(1): 181-188. doi:10.1007/s00280-008-0733-7.

\title{
Phase I trial of weekly trabectedin (ET-743) and gemcitabine in patients with advanced solid tumors
}

\author{
Wells A. Messersmith, \\ University of Colorado Cancer Center, P.O. Box 6511, Mail Stop 8117, Aurora, CO 80045, USA, \\ wells.messersmith@uchsc.edu
}

Antonio Jimeno,

Sidney Kimmel Comprehensive Cancer Center at Johns Hopkins, Baltimore, MD, USA

David Ettinger,

Sidney Kimmel Comprehensive Cancer Center at Johns Hopkins, Baltimore, MD, USA

Dan Laheru,

Sidney Kimmel Comprehensive Cancer Center at Johns Hopkins, Baltimore, MD, USA

Julie Brahmer,

Sidney Kimmel Comprehensive Cancer Center at Johns Hopkins, Baltimore, MD, USA

Dina Lansey,

Sidney Kimmel Comprehensive Cancer Center at Johns Hopkins, Baltimore, MD, USA

Yasmin Khan,

Sidney Kimmel Comprehensive Cancer Center at Johns Hopkins, Baltimore, MD, USA

Ross C. Donehower,

Sidney Kimmel Comprehensive Cancer Center at Johns Hopkins, Baltimore, MD, USA

Yusri Elsayed,

Johnson \& Johnson Pharmaceutical Research \& Development, L.L.C., Raritan, NJ, USA

Peter Zannikos, and

Johnson \& Johnson Pharmaceutical Research \& Development, L.L.C., Raritan, NJ, USA

Manuel Hidalgo

Sidney Kimmel Comprehensive Cancer Center at Johns Hopkins, Baltimore, MD, USA

\section{Abstract}

Purpose-To determine the maximum tolerated dose (MTD) of trabectedin plus gemcitabine administered on a weekly schedule in patients with advanced solid tumors.

Methods-Patients with ECOG performance status 0-1 and adequate organ function were enrolled. On days 1,8 , and 15 of a 28-day cycle, patients received gemcitabine (starting dose, 800 $\mathrm{mg} / \mathrm{m}^{2}$ ) followed by trabectedin (starting dose, $0.3 \mathrm{mg} / \mathrm{m}^{2}$ ). Strict liver function test treatment criteria were employed to avoid hepatic toxicity seen in previous trabectedin studies. Plasma samples were collected during cycles 1 and 2 for pharmacokinetic analyses.

Results-Fifteen patients received $\geq 1$ dose, with a median of two treatment cycles (range 1-10). The most common drug-related toxicity was hepatic. Dose reductions were required for

(C) Springer-Verlag 2008

Correspondence to: Wells A. Messersmith. 
trabectedin in four (27\%) patients and gemcitabine in six (40\%) patients. Cycle delays/dose holds were required in $11(73 \%)$ patients and doses above trabectedin $0.4 \mathrm{mg} / \mathrm{m}^{2}$ and gemcitabine 1,000 $\mathrm{mg} / \mathrm{m}^{2}$, which is the recommended phase II dose, were not feasible. Seven patients maintained stable disease after two cycles. Gemcitabine and trabectedin pharmacokinetics were not altered substantially with concomitant administration.

Conclusions-Given the lack of pharmacokinetic interaction and potential efficacy of trabectedin and gemcitabine combination therapy, further study is warranted with alternate schedules.

\section{Keywords}

Dose-finding; Phase I; Gemcitabine; Pharmacokinetics; Trabectedin

\section{Introduction}

Most advanced solid tumor malignancies remain incurable, and the search for new cancer therapeutics with novel mechanisms of action has recently extended to marine organisms. Trabectedin (YONDELIS ${ }^{\circledR}$, ET-743; Johnson \& Johnson Pharmaceutical Research \& Development, L.L.C., Raritan, NJ, USA; PharmaMar S.A.U., Madrid, Spain) is a tetrahydroisoquinolone alkaloid derivative originally isolated from the marine ascidian Ecteinascidia turbinata (sea squirt) and now produced synthetically. Trabectedin has a complex mechanism of action, involving DNA binding [25, 27], interference with transcription factor binding [24], and activation of the transcription-coupled nucleotide excision repair (NER) system to create lethal single-strand breaks in DNA [4, 35]. The cytotoxicity of trabectedin has been demonstrated in several in vitro and preclinical studies involving a variety of cancer types [12, 14, 18, 29, 37].

In clinical trials, single-agent trabectedin in a variety of administration schedules has achieved clinical responses in patients with a variety of tumor types, including ovarian cancer, osteosarcoma, soft tissue sarcoma, and breast cancer [7, 8, 11, 16, 17, 21, 30, 39, 40]. Recent data suggest that single-agent trabectedin may become a standard of care in advanced pre-treated sarcomas, with clinical benefit observed in patients with liposarcoma and leiomyosarcoma following failure of all conventional treatment options [26]. Moreover, trabectedin was approved in the European Union in September 2007 for treatment of patients with advanced soft tissue sarcoma. The most common side effects have included cytopenias, which have been dose limiting, as well as nonhematologic toxicities such as hepatotoxicity manifesting as elevated transaminases, nausea/vomiting, and malaise. Hepatotoxicity has been reversible, rarely lasting $>7$ days, and noncumulative in patients with strict eligibility criteria including limited increases of total bilirubin, alkaline phosphatase (ALP), and aspartate (AST) and alanine aminotransferase (ALT). The mechanism of the transient transaminitis, or more rare cholangitis, is not known. Experience in patients with compromised liver function is limited to date. Interestingly, liver toxicity has been ameliorated by premedication with steroids in animal models [5]; dexamethasone has been employed as an antiemetic premedication in some phase II studies [7], and dexamethasone co-treatment has been associated with a decreased incidence of severe trabectedin-associated toxicities [28]. Phase I and II studies with trabectedin have evaluated a number of treatment schedules, the most extensive being every 3 weeks given over 3 or 24 $\mathrm{h}$. The results of preclinical studies of trabectedin administered in combination with other agents suggested that additive or synergistic effects may be achieved [22, 33, 34].

Gemcitabine (GEMZAR ${ }^{\circledR}$, Eli Lilly and Company, Indianapolis, IN) is a nucleoside analog that is indicated for the treatment of: pancreatic cancer; non-small cell lung cancer (NSCLC), in combination with cisplatin; advanced ovarian cancer, in combination with 
carboplatin; and breast cancer, in combination with paclitaxel; and has shown promising efficacy either alone or combined with taxanes in patients with chemotherapy-resistant soft tissue sarcomas $[13,19,20,23]$. Because trabectedin and gemcitabine act by different mechanisms, have activity against a variety of solid tumor types including soft tissue sarcomas [10], and are administered on weekly dosing schedules, it was of interest to study them in combination for the treatment of patients with advanced malignancies.

The primary objective of this study was to determine the maximum tolerated dose (MTD) of trabectedin administered in combination with gemcitabine once weekly for three consecutive weeks in a 4-week cycle. Secondary objectives included evaluating the safety and pharmacokinetics of the trabectedin/gemcitabine combination.

\section{Methods}

Patients

Patients $\geq 18$ years of age with histologically confirmed advanced solid tumor malignancies refractory to standard treatment options and/or for whom a gemcitabine-based regimen was appropriate were eligible for enrollment. Patients were required to have: an Eastern Cooperative Oncology Group (ECOG) performance status of 0 or 1; adequate hematologic function (hemoglobin $\geq 9 \mathrm{~g} / \mathrm{dL}$, absolute neutrophil count $[\mathrm{ANC}] \geq 1,500 / \mu \mathrm{L}$, platelets $\geq$ $100,000 / \mu \mathrm{L}$ ); adequate hepatic function (serum total bilirubin $\leq$ upper limit of normal [ULN], serum total ALP $\leq \mathrm{ULN}$ [or if elevated, ALP liver fraction or $5^{\prime}$ nucleotidase $\leq$ ULN], serum AST and ALT $\leq \mathrm{ULN}$, serum albumin $\geq 2.5 \mathrm{~g} / \mathrm{dL}$ ); and adequate renal function (serum creatinine $\leq \mathrm{ULN}$ ). Patients were excluded if they had prior exposure to trabectedin; known hypersensitivity to dexamethasone or components of trabectedin or gemcitabine; known central nervous system metastasis; peripheral neuropathy zgrade 2; were pregnant or breastfeeding or of reproductive potential (males or females) and not employing adequate contraception; had other serious concomitant illness; were using concomitant medications that decrease hepatic blood flow, such as somatostatin and its analogs, octreotide and lanreotide and non-cardioselective beta-blockers. A minimum washout of 4 weeks since prior radiation therapy, hormonal therapy, biological therapy, therapy with any investigational agent or chemotherapy (6 weeks if the regimen included nitrosureas or mitomycin C) was required.

\section{Study design}

This phase I, open-label, dose-finding combination study was conducted at a single site (Sidney Kimmel Comprehensive Cancer Center at Johns Hopkins, Baltimore, MD, USA). It included a screening visit a treatment phase, a treatment termination visit, and a follow-up phase of at least 30 days after treatment termination. The clinical protocol was approved by the Johns Hopkins Institutional Review Board, and all patients provided written informed consent prior to study drug administration.

\section{Drug dosage and administration}

Trabectedin and gemcitabine were administered by intravenous (IV) infusion on days 1,8 and 15 of a 28-day cycle. Patients were premedicated with IV dexamethasone $10 \mathrm{mg}$ prior to gemcitabine administration. Gemcitabine was given as a 30-min peripheral or central infusion, followed $30 \mathrm{~min}$ later by a 3 -h central infusion of trabectedin. Planned dose levels were trabectedin $0.3,0.4,0.475,0.535$ and $0.58 \mathrm{mg} / \mathrm{m}^{2}$ and gemcitabine 800 and $1,000 \mathrm{mg} /$ $\mathrm{m}^{2}$. A standard phase I " $3+3$ " dose escalation design was used, with the recommended dose for phase II testing defined as the highest dose level at which no more than 1 of 6 patients experienced dose-limiting toxicity (DLT). No intrapatient dose escalation was allowed. Use 
of colony-stimulating factor (CSF) according to institutional and American Society of Clinical Oncology (ASCO) guidelines was permitted after cycle 1.

\section{Safety}

Adverse events (AEs) were graded using the National Cancer Institute Common Toxicity Criteria (NCI-CTC), version 2.0. DLT was defined as any of the following during the first cycle: grade 4 neutropenia (ANC $<500 / \mu \mathrm{L}$ ) for $>5$ days; febrile neutropenia (ANC $<500 /$ $\mu \mathrm{L}$ with fever [body temperature $\geq 38.5^{\circ} \mathrm{C}$ ] or sepsis); thrombocytopenia (platelets < $25,000 / \mu \mathrm{L}$ ); any grade $\geq 3$ nonhematologic toxicity (except nausea/vomiting and grade 3 transaminitis lasting <1 week); or delay of continuation of therapy $>3$ weeks. Dose reductions were not considered as DLTs.

Study drugs were administered if the following criteria for continuation of treatment were met: on day 1 of each cycle, ANC $\geq 1,500 / \mu \mathrm{L}$, platelets $\geq 100,000 / \mu \mathrm{L}$, total bilirubin $\leq$ ULN, and transaminases, creatine phosphokinase (CPK) and other nonhematologic drugrelated effects < grade 2; on days 8 and 15 of each cycle, same as above, except ANC $\geq$ $1,000 / \mu \mathrm{L}$ and platelets $275,000 / \mu \mathrm{L}$. If day 1 criteria were not met, the next dose of study drugs was delayed for 1 week and re-evaluated weekly; if day 8 or 15 criteria were not met, study drugs were not administered for that day. Missed day 8 or 15 doses were not substituted. A missed dose was designated a dose hold. A maximal cycle delay of 3 weeks was allowed for recovery from toxicity. If recovery took $>3$ weeks, the patient was discontinued from the protocol. Treatment continued until attaining unacceptable toxicity, disease progression or withdrawal of consent.

Dose reductions to the previous dose level for both agents were instituted for DLT in cycle 2 or later, as well as for total bilirubin > ULN at any time. No more than two dose reductions were allowed for each drug. The initial protocol included dose reductions for ALP > ULN and grade $\geq 3$ ALT/AST elevation (patients did not receive study drug unless they had recovery to grade 1). Patients were to be discontinued from the study for DLT and if grade $\geq$ 3 ALT/AST elevations did not recover to grade 1 within 3 weeks. The protocol was subsequently amended (during dose level 1 ) so that dose reductions were not necessary for a grade 1 elevation of ALP nor transient ( $<1$ week) grade 3 elevations of ALT/AST.

\section{Assessments}

AEs and toxicity were assessed weekly. Treatment responses were determined clinically and radiologically after every two cycles of therapy based on the Response Evaluation Criteria in Solid Tumors (RECIST) guidelines [36].

Baseline evaluations were conducted within 14 days of study entry and included medical history, concurrent medications, vital signs, physical exam, urine pregnancy test (if applicable), electrocardiogram, complete blood count (CBC) with differential, and serum chemistry including albumin, ALP (liver fraction or $5^{\prime}$ nucleotidase if ALP > ULN), total bilirubin, ALT, AST, CPK and total protein. Radiologic evaluations were performed within 4 weeks of study entry. Vital signs were recorded on each treatment day, and CBCs and serum chemistries including liver function tests were performed weekly. Physical exams were repeated once per cycle. An off-study follow-up evaluation of toxicities and blood tests was performed.

\section{Drug assay and pharmacokinetic analysis}

Serial plasma samples for pharmacokinetic analyses were collected during cycles 1 and 2 at the following time points: immediately prior to treatment with gemcitabine, and at $20 \mathrm{~min}$, $1,1.5,3$, and $3 \mathrm{~h} ; 50 \mathrm{~min}, 5,6$, and $9 \mathrm{~h}$ after the start of the gemcitabine infusion. The 
concentration of trabectedin was measured in plasma samples collected prior to dosing and at $30 \mathrm{~min}, 2$, and $3 \mathrm{~h}, 50 \mathrm{~min}, 4,5,8,11,27,51$, and $168 \mathrm{~h}$ after the start of the trabectedin infusion. On cycle 1 days 8 and 15, and cycle 2 days 1, 8, and 15, samples were collected pre-dose, at $20 \mathrm{~min}$ (10 min prior to the end of the gemcitabine infusion), and $200 \mathrm{~min}$ (10 min prior to the end of the trabectedin infusion).

Plasma concentrations of trabectedin were determined with a validated liquid chromatography/mass spectrometry/mass spectrometry (LC-MS/MS) method with a calibration range of $0.025-2.5 \mathrm{ng} / \mathrm{mL}$. Gemcitabine and $2^{\prime}$-deoxy- $2^{\prime}, 2^{\prime}$-difluorouridine $(\mathrm{dFdU})$ plasma concentrations were quantified by a validated high performance liquid chromatography ultraviolet (HPLC-UV) method. The validated concentration range was 0.6-20 $\mu \mathrm{g} / \mathrm{mL}$ and 1.35-90 $\mu \mathrm{g} / \mathrm{mL}$ for gemcitabine and $\mathrm{dFdU}$, respectively. For both assays, quality control samples spiked at three concentration levels in duplicate were used for batch acceptance criteria. At least four out of six quality control samples had to quantitate within $15 \%$ of nominal for a batch to be acceptable. All samples were analyzed at the bioanalytical laboratory of the Slotervaart hospital in Amsterdam, The Netherlands.

Pharmacokinetic parameters were to be estimated for trabectedin, gemcitabine, and $\mathrm{dFdU}$ using conventional noncompartmental methods, as implemented in WinNonlin, Version 5.1 (Pharsight).

\section{Statistical considerations}

All patients who received $\geq 1$ dose of each study drug were included in the toxicity and efficacy analyses. For the pharmacokinetic analyses, parameters are presented as mean values $\pm \mathrm{SD}$, and for all tests, the a priori cutoff for statistical significance was $P<0.05$. Differences between pharmacokinetic parameters were compared by a paired Student's $t$ test. Statistical calculations were performed with the Statistical Analysis System, Version 8.2 (SAS Institute, Cary, NC, USA).

\section{Results}

\section{Patient population}

This trial was conducted between March 2003 and April 2005. Fifteen patients received $\geq 1$ dose of study medication and were included in the analysis population. The majority of patients in the analysis population had sarcoma $(n=5)$ or NSCLC $(n=3)$, and $67 \%$ had an ECOG performance status of 1 . Relevant pretreatment characteristics of the analysis population are summarized in Table 1 . Over half $(10 / 15 ; 67 \%)$ of the patients terminated the study by the end of cycle 2 ; for the five who did not, terminations were at the end of cycles 3 $(n=1), 4(n=1), 6(n=2)$, and $10(n=1)$. Reasons for termination were disease progression $(n=8)$, AE $[n=5 ; 2$ of which were considered possibly drug related (1 patient with nausea and peripheral edema and 1 with nausea and dehydration)], death $(n=1$, a suicide deemed to be unrelated), and patient choice $(n=1)$.

\section{Safety}

The study was terminated due to an unacceptable frequency of adjustments to the weekly dosing schedule for hepatic toxicity. Only dose levels 1, 2, and 3 were evaluated. In all, 45 4-week cycles were administered. The median number of cycles was 2 (range 1-10), with median cumulative doses of trabectedin $1.79 \mathrm{mg} / \mathrm{m}^{2}$ and gemcitabine $4,592 \mathrm{mg} / \mathrm{m}^{2}$. Across the three dose cohorts, median cumulative dose intensity was $0.802 \mathrm{mg} / \mathrm{m}^{2} / 4$ weeks for trabectedin and $1841.02 \mathrm{mg} / \mathrm{m}^{2} / 4$ weeks for gemcitabine. Median relative dose intensity (actual/planned dose intensity) over all reported cycles ranged from $70 \%$ trabectedin and $67 \%$ gemcitabine at dose level 3 to 77 and $76 \%$, respectively, at dose level 1 . Dose 
reductions were required for trabectedin in four (27\%) patients (all in cycle 2, with one patient requiring a second dose reduction in cycle 3 ) and for gemcitabine in six (40\%) patients (five in cycle 2 and one in cycle 3 ). Cycle delays and dose holds due to AEs were required for $11(73 \%)$ patients, predominantly within the first two cycles, and were most often related to liver toxicity.

No DLTs were seen in any of the cohorts (Table 2). Dose levels 1 and 3 were expanded to six patients due to cycle delays or dose reductions required in the first three patients. Since four of six patients at dose level 3 required dose holds/cycle delays (two required dose reductions), no further dose escalation was performed. Of the 25 total drug-related dose holds or reductions, $17(68 \%)$ were due to elevated AST/ALT, 2 (8\%) to elevated ALP, 4 (16\%) to thrombocytopenia, and $1(4 \%)$ each to congestive heart failure and pulmonary edema. The most frequently reported grade $3 / 4$ drug-related AE was ALT increase (33\%) (Table 3). Although a high frequency of hepatic toxicity was observed, no patient had cholestasis or liver failure. The most common drug-related AEs of any severity were ALT increase (80\%), fatigue (73\%), nausea (67\%), AST increase (60\%), vomiting (40\%), neutropenia (33\%) and anorexia (27\%).

\section{Efficacy}

There were no complete or partial responses to therapy in this heavily pretreated patient population. Seven $(47 \%)$ patients maintained stable disease for a duration of 56-226 days: $\operatorname{NSCLC}(n=3)$; colorectal cancer $(n=2)$; renal cancer and sarcoma ( $n=1$ each). Two of these seven patients maintained stable disease for at least 6 months.

\section{Pharmacokinetic analysis}

The pharmacokinetics of trabectedin in the present study were similar to those observed previously. Maximum plasma concentrations of trabectedin were typically observed between 2 and $3 \mathrm{~h}$ after the start of the 3-h infusion. Upon cessation of the infusion, the concentrations declined in a multiexponential manner. Trabectedin exhibited a high plasma clearance and relatively large distribution volume across the dose groups (Table 4). The mean terminal half-life of trabectedin in plasma ranged from 32.7 to $43.4 \mathrm{~h}$. Interpatient variability in the plasma and $C_{\max } \mathrm{AUC}_{\infty}$ values of trabectedin for the three treatment groups ranged from 14.8 to $118 \%$ (expressed as coefficient of variation). Plasma concentrations of gemcitabine (and dFdU) were not reported for dose level 1, because the plasma samples were inadvertently collected in tubes that did not contain stabilizer, and were thus deemed unreliable. For gemcitabine, most samples collected from the second and third dose levels at $1 \mathrm{~h}$ after the end of infusion or later contained concentrations of gemcitabine that were below the limits of detection; therefore, plasma pharmacokinetic parameters of gemcitabine could not be calculated. Individual gemcitabine concentrations at 20 min after the start of the first dose in cycle 1 ranged from 2.88 to $20.5 \mu \mathrm{g} / \mathrm{mL}$ and from 7.03 to $21.9 \mu \mathrm{g} / \mathrm{mL}$ when gemcitabine 800 and $1,000 \mathrm{mg} / \mathrm{m}^{2}$, respectively, was coadministered with trabectedin $0.4 \mathrm{mg} / \mathrm{m}^{2}$. Maximum plasma concentrations of $\mathrm{dFdU}$ were typically observed at approximately $0.3-1 \mathrm{~h}$ after the start of the 30-min infusion (Table 4). Upon cessation of the infusion, the concentrations declined in a monoexponential manner. The mean terminal half-life values of dFdU in plasma were 6.82 and $4.79 \mathrm{~h}$, respectively. Interpatient variability in the plasma $C_{\max }$ and $\mathrm{AUC}_{\infty}$ values of $\mathrm{dFdU}$ ranged from 11.4 to $61.3 \%$ (expressed as coefficient of variation).

\section{Discussion}

In this phase I study, the combination of trabectedin and gemcitabine was evaluated to determine the MTD in patients with advanced malignancies. Due to hepatic toxicity 
observed in earlier trabectedin studies, strict liver function test criteria for treatment were implemented in this trial. Although no DLTs were observed in cycle 1 at any of the dose levels explored, a high frequency of dose adjustments (cycle delays, dose holds and dose reductions) in the weekly schedule precluded realistic determination of the MTD. The protocol was amended during exploration of the first dose level to liberalize the liver function test criteria, but further changes were not feasible due to safety concerns.

The pharmacokinetic parameters of trabectedin and gemcitabine were similar to those reported previously for single-agent studies of both drugs. However, due to the relatively small number of patients with available data, definitive conclusions regarding drug interactions could not be made. Efficacy of the trabectedin/gemcitabine combination, although not a primary endpoint of the study, was promising in that almost half (47\%) of the patients analyzed maintained stable disease after two cycles.

In addition to its efficacy in approved combinations with cisplatin, carboplatin and paclitaxel, gemcitabine has shown activity and tolerability in phase I trials when given in combination with topotecan [32] and docetaxel [31]. Gemcitabine has also shown activity in phase II trials as a single agent and in combination with docetaxel in patients with advanced sarcomas, particularly leiomyosarcoma [13, 19, 20, 23]. Trabectedin has demonstrated efficacy as a single agent in patients with sarcoma [7, 8, 17, 26, 39] and has shown promise in phase I trials against a variety of malignancies when given in combination with capecitabine [9], doxorubicin [1] or pegylated liposomal doxorubicin [3], but nearly all previous studies have used an every-3-week schedule. Although dose level 3 contained an approved gemcitabine dose of $1,000 \mathrm{mg} / \mathrm{m}^{2}$ per week, the highest dose of trabectedin evaluated in combination with this gemcitabine dose was $0.4 \mathrm{mg} / \mathrm{m}^{2}$ per week. This is slightly less than a third of the recommended dose of single-agent trabectedin as a 3-h infusion in 3 -week cycles $\left(1.3 \mathrm{mg} / \mathrm{m}^{2}\right)[11,30]$. A single-agent study of trabectedin using a weekly schedule similar to this trial has been presented in abstract form, with a recommended dose of $0.610 \mathrm{mg} / \mathrm{m}^{2}$ [2]. In that study, grade $\geq 3 \mathrm{AST} / \mathrm{ALT}$ elevations and grade $\geq 3$ neutropenia were seen at all dose levels above 0.610 and $0.580 \mathrm{mg} / \mathrm{m}^{2}$, respectively. It is possible that combination weekly schedules of trabectedin will be problematic due to hepatotoxicity, albeit transient and reversible, which prevents regular dosing. Moreover, the toxicity profiles of trabectedin and gemcitabine overlap, in particular with regard to myelosuppression and increases in transaminases. Trabectedin has also been tested in a daily $\times 5$ schedule every 3 weeks [38], where the MTD was $0.325 \mathrm{mg} / \mathrm{m}^{2}$ due to cytopenias. Interestingly, in that study hepatotoxicity was common in doses $>0.216 \mathrm{mg} / \mathrm{m}^{2}$, but this was not dose-limiting. A recently published pharmacokinetic/pharmacodynamic model of trabectidin using data from 711 patients in 14 studies with trabectidin confirmed the liver-protective effects of dexamethasone premedication, and there were no major differences between 3- and 24-h infusions [6].

Future studies of this combination could involve exploration of alterative dosing schedules, such as trabectedin day 1 and gemcitabine days 1 and 8 every 21 days. It should be noted that gemcitabine $2,000 \mathrm{mg} / \mathrm{m}^{2}$ has been dosed every 3 weeks in a combination trial with cisplatin for NSCLC [15], so an every-21-day schedule could be considered, but cytopenias, hepatotoxicity and other side effects may be prohibitive.

In conclusion, this study did not identify a DLT-defined recommended phase II dose, but rather a weekly feasible dose of trabectedin in combination with gemcitabine. The results obtained suggest a potentially manageable toxicity profile and no evidence of a significant pharmacokinetic interaction with the combination of trabectedin and gemcitabine for the treatment of advanced malignancies. This combination is particularly appealing for phase II testing in patients with soft tissue sarcomas in whom the two drugs as single agents have 
clinical activity. However, it must be noted that the dose of trabectedin that is tolerable is lower than that used as a single agent. Further study using different schedules may be warranted. This could include the 3-weekly dosing that is shown to be effective in soft tissue sarcoma.

\section{Acknowledgments}

This clinical trial (Study ET-743-USA-7) was sponsored by Johnson \& Johnson Pharmaceutical Research \& Development, L.L.C., Raritan, NJ and PharmaMar S.A.U., Madrid, Spain. We would like to thank Tom Verhaeghe for overseeing the bioanalysis of trabectedin and gemcitabine. We would also like to thank Lisa Shannon, PharmD, of Scientific Connexions, for providing medical co-writing and editing services.

\section{References}

1. Blay JY, von Mehren M, Samuels BL, et al. Combination of trabectedin (T) and doxorubicin (D) for the treatment of patients with soft tissue sarcoma (STS): safety and efficacy analysis. J Clin Oncol. 2007; 25:18s. Abstr 10078.

2. Chu, QSC.; Schwartz, G.; Forouzesh, B., et al. Phase I and pharmacokinetic (PK) study of trabectedin (ET-743) administered as a 1-hour infusion weekly for 3 consecutive weeks every 4 weeks to patients with advanced cancer. Presented at the 2004 AACR-NCI-EORTC International Conference on Molecular Targets and Cancer Therapeutics; 28 September-1 October; Geneva, Switzerland. 2004.

3. Cohen RB, Schilder RJ, Cheng J, et al. Final results of a combination study between trabectedin and pegylated liposomal doxorubicin (PLD) in patients with advanced malignancies. J Clin Oncol. 2005; 23:16s. Abstract 3074.

4. Damia G, Silvestri S, Carrassa L, et al. Unique pattern of ET-743 activity in different cellular systems with defined deficiencies in DNA-repair pathways. Int J Cancer. 2001; 92:583-588. [PubMed: 11304695]

5. Donald S, Verschoyle RD, Greaves P, et al. Complete protection by high-dose dexamethasone against the hepatotoxicity of the novel antitumor drug Yondelis (ET-743) in the rat. Cancer Res. 2003; 63:5902-5908. [PubMed: 14522916]

6. Fetterly GJ, Owen JS, Stuychens K, et al. Semimechanistic pharmacokinetic/pharmacodynamic model for hepatoprotective effect of dexamethasone on transient transaminitis after trabectidin (ET-743) treatment. Cancer Chemother Phamacol. 2007 Oct 9. Epub ahead of print.

7. Garcia-Carbonero R, Supko JG, Maki RG, et al. Ecteinascidin-743 for chemotherapy-naïve patients with advanced soft tissue sarcomas: multicenter phase II and pharmacokinetic study. J Clin Oncol. 2005; 23:5484-5492. [PubMed: 16110008]

8. Garcia-Carbonero R, Supko JG, Manola J, et al. Phase II and pharmacokinetic study of ecteinascidin 743 in patients with progressive sarcomas of soft tissues refractory to chemotherapy. J Clin Oncol. 2004; 22:1480-1490. [PubMed: 15084621]

9. Gore L, Rivera E, Laffallee K, et al. Phase I combination study of trabectedin and capecitabine in patients with advanced malignancies. J Clin Oncol. 2006; 24:18s. Abstract 2079.

10. Grosso F, Jones RL, Demetri GD, et al. Efficacy of trabectedin (ecteinascidin-743) in advanced pretreated myxoid liposarcomas: a retrospective study. Lancet Oncol. 2007; 8:595-602. [PubMed: 17586092]

11. Gurtler JS, Goldstein L, Delprete S, et al. Trabectedin in third line breast cancer: a multicenter, randomized, phase II study comparing two administration regimens. J Clin Oncol. 2005; 23:16s. Abstract 625.

12. Hendriks HR, Fiebig HH, Giavazzi R, et al. High antitumor activity of ET743 against human tumour xenografts from melanoma, non-small-cell lung and ovarian cancer. Ann Oncol. 1999; 10:1233-1240. [PubMed: 10586342]

13. Hensley ML, Maki R, Venkatraman E, et al. Gemcitabine and docetaxel in patients with unresectable leiomyosarcoma: results of a phase II trial. J Clin Oncol. 2002; 20:2824-2831. [PubMed: 12065559] 
14. Izbicka E, Lawrence R, Raymond E, et al. In vitro antitumor activity of the novel marine agent, ecteinascidin-743 (ET-743, NSC-648766) against human tumors explanted from patients. Ann Oncol. 1998; 9:981-987. [PubMed: 9818072]

15. Karacetin D, Incekara O. Gemcitabine-cisplatin given on day 1 every 3 weeks in advanced nonsmall cell lung cancer. J BUON. 2006; 11:181-184. [PubMed: 17318968]

16. Laverdiere C, Kolb EA, Supko JG, et al. Phase II study of ecteinascidin 743 in heavily pretreated patients with recurrent osteosarcoma. Cancer. 2003; 98:832-840. [PubMed: 12910529]

17. Le Cesne A, Blay JY, Judson I, et al. Phase II study of ET-743 in advanced soft tissue sarcomas: a European Organisation for the Research and Treatment of Cancer (EORTC) Soft Tissue and Bone Sarcoma Group Trial. J Clin Oncol. 2005; 23:576-584. [PubMed: 15659504]

18. Li WW, Takahashi N, Jhanwar S, et al. Sensitivity of soft tissue sarcoma cell lines to chemotherapeutic agents: identification of ecteinascidin-743 as a potent cytotoxic agent. Clin Cancer Res. 2001; 7:2908-2911. [PubMed: 11555609]

19. Look KY, Sandler A, Blessing JA, et al. Phase II trial of gemcitabine as second-line chemotherapy of uterine leiomyosarcoma: a Gynecologic Oncology Group (GOG) Study. Gynecol Oncol. 2004; 92:644-647. [PubMed: 14766260]

20. Maki RG, Wathen JK, Patel SR, et al. Randomized phase II study of gemcitabine and docetaxel compared with gemcitabine alone in patients with metastatic soft tissue sarcomas: results of sarcoma alliance for research through study 002. J Clin Oncol. 2007; 25:2755-2763. [PubMed: 17602081]

21. McMeekin DS, Krasner C, Chan S, et al. Final results of a phase II study of weekly trabectedin in second/third line ovarian carcinoma. J Clin Oncol. 2005; 23:16s. Abstract 5011.

22. Meco D, Colombo T, Ubezio P, et al. Effective combination of ET-743 and doxorubicin in sarcoma: preclinical studies. Cancer Chemother Pharmacol. 2003; 52:131-138. [PubMed: 12783202]

23. Merimsky O, Meller I, Flusser G, et al. Gemcitabine in soft tissue or bone sarcoma resistant to standard chemotherapy: a phase II trial. Cancer Chemother Pharmacol. 2000; 45:177-181. [PubMed: 10663634]

24. Minuzzo M, Marchini S, Broggini M, et al. Interference of transcriptional activation by the antineoplastic drug ecteinascidin-743. Proc Natl Acad Sci. 2000; 97:6780-6784. [PubMed: 10841573]

25. Moore BM, Seaman FC, Wheelhouse RT, et al. Mechanism for the catalytic activation of ecteinascidin 743 and its subsequent alkylation of guanine N2. J Am Chem Soc. 1998; 120:24902491.

26. Morgan JA, Le Cesne A, Chawla S, et al. Randomized phase II study of trabectedin in patients with liposarcoma and leiomyosarcoma(L-sarcomas) after failure of prior anthracyclines (A) and ifosfamide (I). J Clin Oncol. 2007; 25:18s. Abstract 10060.

27. Pommier Y, Kohlhagen G, Bailly C, et al. DNA sequence-and structure-selective alkylation of guanine N2 in the DNA minor groove by ecteinascidin 743, a potent antitumor compound from the Caribbean tunicate Ecteinascidia turbinata. Biochemistry. 1996; 35:13303-13309. [PubMed: 8873596]

28. Puchalski TA, Ryan DP, Garcia-Carbonero R, et al. Pharmacokinetics of ecteinascidin 743 administered as a 24-h continuous intravenous infusion to adult patients with soft tissue sarcomas: associations with clinical characteristics, pathophysiological variables and toxicity. Cancer Chemother Pharmacol. 2002; 50:309-319. [PubMed: 12357306]

29. Scotlandi K, Perdichizzi S, Manara MC, et al. Effectiveness of ecteinascidin-743 against drugsensitive and -resistant bone tumor cells. Clin Cancer Res. 2002; 8:3893-3903. [PubMed: 12473605]

30. Sessa C, DeBraud F, Perotti A, et al. Trabectedin for women with ovarian carcinoma after treatment with platinum and taxanes fails. J Clin Oncol. 2005; 23:1867-1874. [PubMed: 15774779]

31. Spiridonidis CH, Laufman LR, Jones J, et al. Phase I study of docetaxel dose escalation in combination with fixed weekly gemcitabine in patients with advanced malignancies. J Clin Oncol. 1998; 16:3866-3873. [PubMed: 9850032] 
32. Sun W, Stevenson JP, Gallagher M, et al. A phase I trial of topotecan and gemcitabine administered weekly for 3 consecutive weeks to patients with advanced tumors. Cancer. 2001; 92:414-419. [PubMed: 11466697]

33. Takahashi N, Li WW, Banerjee D, et al. Sequence-dependent enhancement of cytotoxicity produced by ecteinascidin 743 (ET-743) with doxorubicin or paclitaxel in soft tissue sarcoma cells. Clin Cancer Res. 2001; 7:3251-3257. [PubMed: 11595721]

34. Takahashi N, Li WW, Banerjee D, et al. Sequence-dependent synergistic cytotoxicity of ecteinascidin-743 and paclitaxel in human breast cancer cell lines in vitro and in vivo. Cancer Res. 2002; 62:6909-6915. [PubMed: 12460906]

35. Takebayashi Y, Pourquier P, Zimonjic DB, et al. Antiproliferative acitivty of ecteinascidin 743 is dependent upon transcription-coupled nucleotide-excision repair. Nat Med. 2001; 7:961-966. [PubMed: 11479630]

36. Therasse P, Arbuck SG, Eisenhauer EA, et al. New guidelines to evaluate the response to treatment in solid tumors. J Natl Cancer Inst. 2000; 92:205-216. [PubMed: 10655437]

37. Valoti G, Nicoletti MI, Pellegrino A, et al. Ecteinascidin-743, a new marine natural product with potent antitumor activity on human ovarian carcinoma xenografts. Clin Cancer Res. 1998; 4:19771983. [PubMed: 9717828]

38. Villalona-Calero MA, Eckhardt SG, Weiss G, et al. A phase I and pharmacokinetic study of ecteinascidin- 743 on a daily $\times 5$ schedule in patients with solid malignancies. Clin Cancer Res. 2002; 8:75-85. [PubMed: 11801542]

39. Yovine A, Riofrio M, Blay JY, et al. Phase II study of ecteinascidin-743 in advanced pretreated soft tissue sarcoma patients. J Clin Oncol. 2004; 22:890-899. [PubMed: 14990645]

40. Zelek L, Yovine A, Brain E, et al. A phase II study of Yondelis ${ }^{\circledR}$ (trabectedin, ET-743) as a 24-h continuous intravenous infusion in pretreated advanced breast cancer. Br J Cancer. 2006; 94:16101614. [PubMed: 16736024] 


\section{Table 1}

Demographic and clinical characteristics of the analysis population

\begin{tabular}{ll}
\hline & Total $(\boldsymbol{N}=\mathbf{1 5})$ \\
\hline Sex & \\
Female & 11 \\
Male & 4 \\
Age & \\
18 to 65 & 11 \\
$>$ 65 & 4 \\
Median & 61.0 \\
Range & $26-78$ \\
ECOG performance status \\
0 \\
1 \\
Histology & 5 \\
Sarcoma & 5 \\
Leiomyosarcoma & 3 \\
Other & 2 \\
NSCLC & 3 \\
Colorectal & 2 \\
Renal & 2 \\
Anal & 1 \\
Salivary gland & 1 \\
Endometrial & 1 \\
Prior treatment & \\
Chemotherapy & \\
1 regimen & 2 \\
2 regimens & 4 \\
$>2$ regimens & 8 \\
Radiotherapy & 4 \\
\hline Hormone therapy & 3 \\
\hline I & \\
\hline
\end{tabular}

ECOG Eastern Cooperative Oncology Group, NSCLC non-small cell lung cancer 


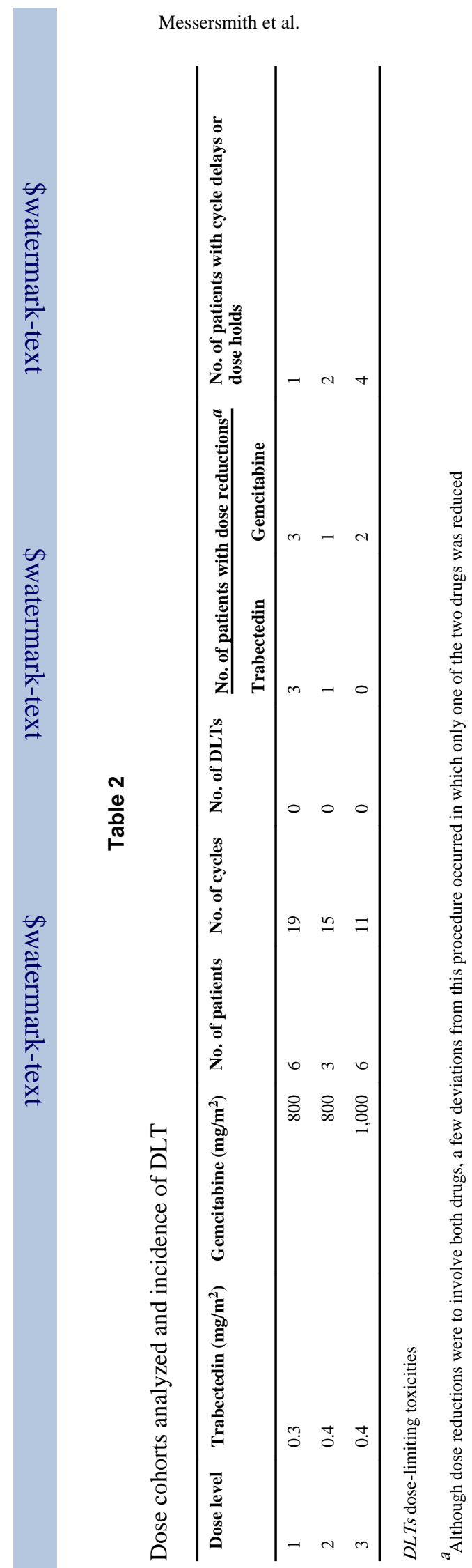

Page 12 . 
Table 3

Grade 3/4 drug-related adverse events

\begin{tabular}{|c|c|c|c|c|}
\hline & $\begin{array}{l}\text { Dose level } 10.3 / 800 \mathrm{mg} / \mathrm{m}^{2} \\
\text { trabectedin/gemcitabine }(n \\
=6)\end{array}$ & $\begin{array}{l}\text { Dose level } 20.4 / 800 \mathrm{mg} / \mathrm{m}^{2} \\
\text { trabectedin/gemcitabine ( } n \\
=3)\end{array}$ & $\begin{array}{l}\text { Dose level } 30.4 / 1000 \mathrm{mg} / \mathrm{m}^{2} \\
\text { trabectedin/gemcitabine ( } n \\
=6 \text { ) }\end{array}$ & $\begin{array}{l}\text { Total }[N=15 \\
(\%)]\end{array}$ \\
\hline Total with grade $3 / 4 \mathrm{AE}$ & 3 & 2 & 3 & $8(53)$ \\
\hline ALT increased & 1 & 2 & 2 & $5(33)$ \\
\hline Vomiting & 2 & 1 & 1 & $4(27)$ \\
\hline Neutropenia & 1 & 2 & 1 & $4(27)$ \\
\hline AST increased & 1 & 1 & 1 & $3(20)$ \\
\hline Weight decreased & 1 & 0 & 0 & $1(7)$ \\
\hline Cardiac failure & 1 & 0 & 0 & $1(7)$ \\
\hline Acute prerenal failure & 1 & 0 & 0 & $1(7)$ \\
\hline Leukopenia & 0 & 0 & 1 & $1(7)$ \\
\hline Thrombocytopenia & 0 & 0 & 1 & $1(7)$ \\
\hline
\end{tabular}

$A E$ adverse event, $A L T$ alanine aminotransferase, $A S T$ aspartate aminotransferase

Note: AEs reported at any time from first treatment dose to within 30 days of last treatment dose are included. Incidence is based on the number of patients. Drug-related means possible, probable, or very likely. Toxicity grade: NCI Common Toxicity Criteria, version 2.0 


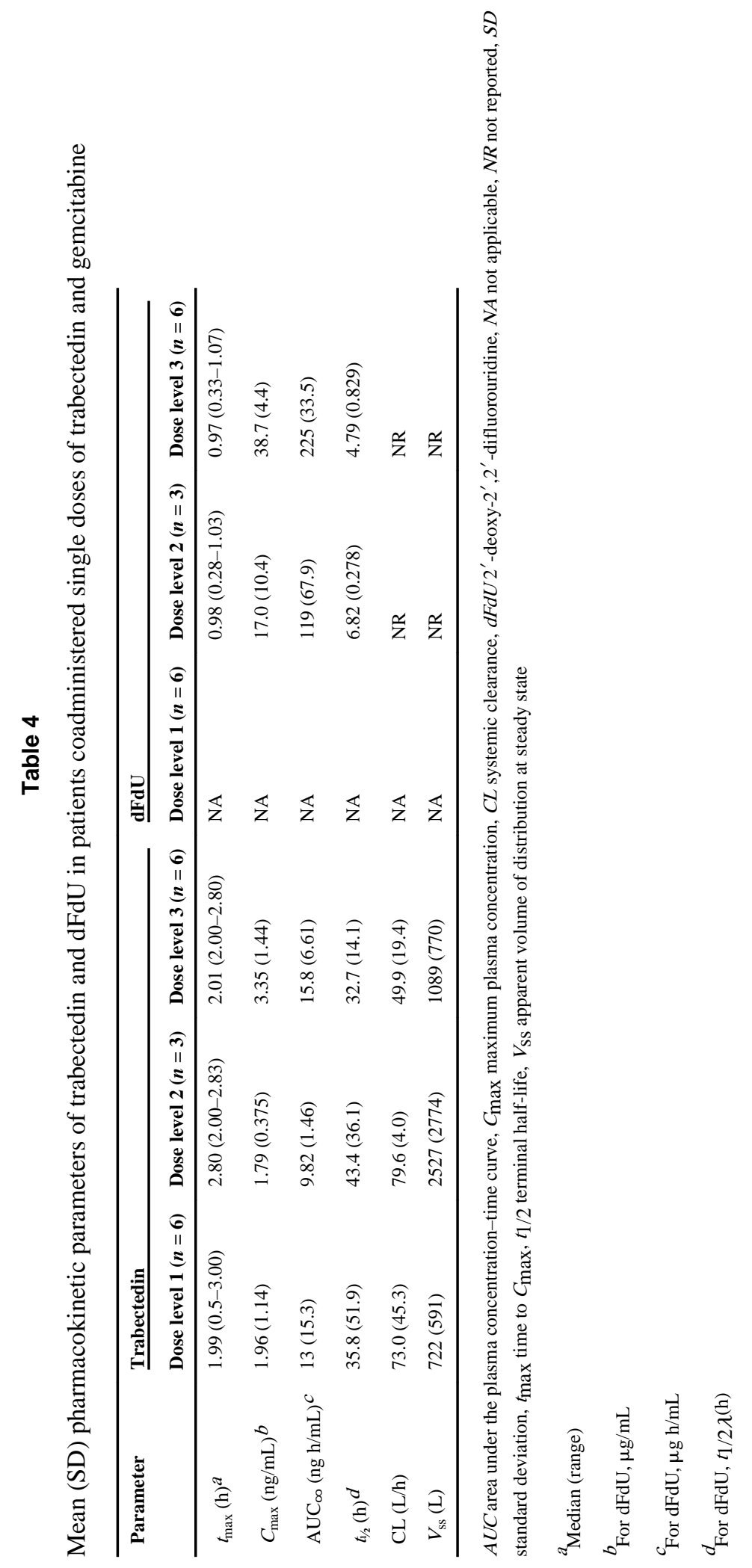

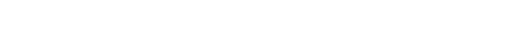

